\title{
Bortezomib Is More Effective to Side Population of RPMI8226 Myeloma Cells than Classical Anti-myeloma Agents
}

\author{
KYOUNG HA KIM ${ }^{1,2}$, HEE-JEONG CHEONG ${ }^{2}$, MIN YOUNG LEE ${ }^{1,2}$, \\ NAMSU LEE ${ }^{1,2}$, KYU-TAEK LEE $^{1}$, SEONG KYU PARK ${ }^{1}$ and JONG-HO WON ${ }^{1,2}$ \\ ${ }^{1}$ Division of Hematology-Oncology, Department of Internal Medicine, and \\ ${ }^{2}$ Institute for Clinical Molecular Biology Research, Soonchunhyang University College of Medicine, \\ Soonchunhyang University Hospital, Seoul, Republic of Korea
}

\begin{abstract}
Aim: Cytotoxic chemotherapy-based treatment of multiple myeloma (MM) is not curative, and the disease eventually recurs. This is partially because although currently available anti-MM strategies are effective in targeting the bulk of tumor cells, they do not target the tumor-initiating subpopulation of cancer stem cells. This study investigated the prevalence and biological functions of side population (SP) cells in MM cell lines including RPMI8226, ARH77, MM.1R and IM 9. Materials and Methods: Flow cytometry-based Hoechst 33342 staining was used to evaluate the existence of SP cells. In addition, the ability of SP cells to regenerate the original population was determined. Results: The frequency of SP cells was heterogeneous. Most cell lines (ARH77, IM9, and MM.lR) contained fewer than 1\% SP cells; however, RPMI8226 contained approximately 10\% SP cells. Sorted SP cells showed a higher proliferative ability and clonogenicity than the MP in the RPMI8226 myeloma cell line. The activity of ATP-binding cassette subfamily $G$ member 2 (ABCG2), which is associated with high rates of proliferation, was higher in SP cells. However, the expression of specific surface markers such as cluster of differentiation (CD)138, CD34, CD38, CD19, CD20, and CD27 did not differ between SP and MP cells. Bortezomib was the only agent that significantly affected proliferation of both SP and MP cells. Conclusion: Our studies demonstrated that the SP fraction of myeloma cells possessed clonogenic tumorinitiating potential and revealed new mechanisms of action for bortezomib on SP cells.
\end{abstract}

Correspondence to: Jong-Ho Won, MD, Ph.D., Professor, Division of Hematology-Oncology, Soonchunhyang University Hospital, 59 Daesagwan-ro, Yongsan-gu, Seoul 04401, Republic of Korea. Tel: +82 27099182/+82 27099203, Fax: +82 27099200, e-mail: jhwon@schmc.ac.kr

Key Words: Multiple myeloma, stem cells, side population, bortezomib.
Multiple myeloma (MM) represents the malignant proliferation of plasma cells derived from a single clone (1). The introduction of novel agents has significantly improved the outcome of patients with MM during the past two decades. However, despite significant advances in treatment, MM remains largely incurable, and extensive research for developing therapeutic approaches for relapsed/refractory MM is required.

The normal counterpart of myeloma cells are terminallydifferentiated plasma cells that lack substantial replicative capacity (2). Hence, it is unclear whether the growth property of myeloma cells is related to clinical development, persistence, and poor prognosis of the disease. Several studies on the recurrence and drug resistance of MM revealed that MM cells include 'cancer stem cells' (3-7). In 2004, Matsui et al. showed that MM stem cells are cluster of differentiation (CD) $138^{-}$B-cells with the ability to replicate and subsequently differentiate into malignant $\mathrm{CD} 138^{+}$plasma cells. In addition, they suggested that $\mathrm{CD} 138^{-}$clonogenic MM cells might be B-cells (3). However, Hosen et al. reported that CD138- clonogenic cells are plasma cells and not B-cells (8). Thus, the precise phenotype of clonogenic cells has not been definitively established.

Side population (SP) cells consist of a large number of cancer-initiating cells (9-12). SP cells have the ability to differentiate into non-SP cells [or main population cells (MP)] both in vitro and in vivo, and are involved in resistance to chemotherapy. In multiple myeloma, SP cells have more prominent clonogenic potential than MP cells (9). Certain studies demonstrated that SP cells had stronger tumorigenicity than MP cells, however, SP cells were not identical to CD138- cells (13-15). Although a number of studies have confirmed the features of MM stem cells, the results of these studies are not consistent.

In this study, we identified the SP cells of MM and evaluated the characteristics of these cells. Furthermore, we investigated whether the SP cells affect drug resistance in MM. 


\section{Materials and Methods}

Culture of MM cell lines. Human MM cell lines RPMI8226, ARH77, and MM.1R were obtained from the American Type Culture Collection (Manassas, VA, USA) and IM 9 was obtained the Korean Cell Line Bank (Cancer Research Institute, Seoul National University, Seoul). The MM cell lines were cultured in Roswell Park Memorial Institute (RPMI)-1640 medium (Gibco, Thermo Fisher Scientific, Waltham MA, USA) supplemented with $10 \%$ fetal bovine serum (FBS; Gibco) and $1 \%$ penicillinstreptomycin (Gibco). Cells were cultured in a humidified incubator with $5 \% \mathrm{CO}_{2}$ at $37^{\circ} \mathrm{C}$.

SP analysis. To identify the SP cells in MM cell lines, the cells were harvested within the passage number of 5 and washed in pre-warmed Hank's balanced salt solution (HBSS; Sigma Aldrich, St. Louis, MO, USA) containing 4\% FBS and $10 \mathrm{mM}$ HEPES (Gibco) buffer and resuspended at a density of $1 \times 10^{6}$ cells $/ \mathrm{ml}$ in HBSS with $4 \%$ FBS and $10 \mathrm{mM}$ HEPES buffer containing $7.5 \mu \mathrm{g} / \mathrm{mI}$ of Hoechst 33342 dye (Invitrogen, Carlsbad, CA, USA). The cells were then incubated for $90 \mathrm{~min}$ at $37^{\circ} \mathrm{C}$ with intermittent shaking. As a negative control, SP cells were preincubated with $50 \mu \mathrm{mol} / \mathrm{I}$ of the ATP binding cassette $(\mathrm{ABC})$ transporter inhibitors reserpine (Sigma Aldrich) or $100 \mu \mathrm{mol} / \mathrm{I}$ verapamil (Sigma Aldrich). At the end of the incubation, the cells were washed and resuspended in ice-cold HBSS with $4 \%$ FBS and $10 \mathrm{mM}$ HEPES buffer. In order to gate only viable cells, propidium iodide solution (PI; Sigma Aldrich) was added to the cells to a final concentration of $2 \mu \mathrm{g} / \mathrm{mI}$. The cells were filtered through a $70-\mu \mathrm{m}$ cell strainer to obtain single-cell suspension. SP and MP cells were then analyzed and sorted using a MOFLO XDP cell sorter (Beckman Coulter, Fullerton, CA, USA). The Hoechst 33342 dye was excited with ultraviolet (UV) laser at $351 \mathrm{~nm}$ and its fluorescence was analyzed at two wavelengths (blue: 405/30 nm; red: 630/30 nm). Cells negative for Hoechst 33342 were included in the SP gate, while cells positive for Hoechst 33342 were collected in the MP gate.

Western blotting. Total protein was extracted from the sorted SP and MP cells using PRO-PREPTM protein extraction solution (iNtRON Biotechnology, Inc., Seongnam-si, Gyeonggi-do, South Korea). The bicinchoninic acid colorimetric method (Pierce, Rockford, IL, USA) was used for determining the protein concentration of the extracts. Equal amounts of protein samples $(30 \mu \mathrm{g})$ were separated by $10 \%$ sodium dodecyl sulfate-polyacrylamide gel electrophoresis (SDSPAGE) and electrophoretically transferred to polyvinylidene difluoride membranes (Bio-Rad, Hercules, CA, USA). The membranes were then blocked with 5\% nonfat dried milk (Bio-Rad) in $50 \mathrm{mM}$ Tris- $\mathrm{HCl}(\mathrm{pH} 7.5), 150 \mathrm{mM} \mathrm{NaCl}$, and $0.1 \%$ (v/v) Tween 20 (TTBS) for 1 hour at room temperature and then incubated with 1:500 dilution of primary antibodies (diluted in 5\% nonfat dried milk/TTBS) overnight at $4^{\circ} \mathrm{C}$ with gentle shaking. Rabbit polyclonal anti-ABCG2 (Cell Signaling Technology, Beverly, MA, USA), rabbit polyclonal anti-octamer-binding transcription factor 4 (OCT4) (Cell Signaling Technology), rabbit polyclonal anti-sex determining region Y-box 2 (SOX2) (Cell Signaling Technology), and rabbit polyclonal anti-NANOG homeobox (NANOG) (Cell Signaling Technology) were used as primary antibodies. $\beta$-Actin was used as an internal control to ensure equal loading of protein samples (mouse monoclonal antibody to $\beta$-actin; Sigma Aldrich). The membranes were then washed with TTBS $(3 \times 15 \mathrm{~min})$ and subsequently incubated with secondary anti-rabbit or anti-mouse horseradish peroxidaseconjugated antibodies (Dako, Glostrup, Denmark) diluted 1:5,000 in $5 \%$ nonfat dried milk/TTBS for $2 \mathrm{~h}$ at room temperature. The membranes were then washed as described above and developed using the enhanced chemiluminescence (ECL) detection system (Amersham, Buckinghamshire, UK). The assay was repeated more than three times to ascertain qualitative reproducibility.

Fluorescence immunophenotyping assay. For analysis of celIsurface antigens, sorted SP and MP cells were washed twice with washing buffer (PBS containing $0.2 \%$ bovine serum albumin and $0.1 \% \mathrm{NaN}_{3}$ ), followed by staining with fluorescein isothiocyanate (FITC) or phycoerythrin (PE)-conjugated mouse antibodies to human CD138, CD38, CD34, CD45, CD19, Ki-67 (BD Biosciences, San Jose, CA, USA), and CD20 (R\&D Systems, Minneapolis, MN, USA) in PBS containing $2 \%$ bovine serum albumin (BSA; Sigma Aldrich) for 30 minutes at $4{ }^{\circ} \mathrm{C}$ in the dark. The labeled cells were washed and resuspended in washing buffer and then analyzed using flow cytometry (FC500; Beckman Coulter).

Cell growth assay. Sorted SP and MP cells $\left(3 \times 10^{5}\right)$ were cultured in RPMI-1640 medium supplemented with $10 \%$ FBS (Gibco) and $1 \%$ penicillin-streptomycin (Gibco). Cell growth of the sorted SP and MP cells was observed after 3, 7, 10, and 14 days of culture using trypan blue (Gibco) exclusion staining. The number of live (unstained) cells was counted using a hemocytometer by light microscopy.

Colony-forming cell assay. Cellular potential clonogenicity of the sorted SP and MP cells was determined using colony-forming cell assay. Briefly, $2 \times 10^{3}$ sorted SP and MP cells were plated separately in $35-\mathrm{mm}$ tissue culture dishes in duplicates in $1.1 \mathrm{mI}$ aliquots of Iscove's modified Dulbecco's medium supplemented with $1.0 \%$ methylcellulose, $30 \%$ FBS, $1 \%$ BSA, $10^{-4}$ M 2-mercaptoethanol, $2 \mathrm{mM}$ L-glutamine, 10\% agar leukocyte-conditioned medium, 3 units/ml recombinant erythropoietin (MethoCultTM; StemCell Technologies, Vancouver, BC, Canada), and a cocktail of growth factors $(10 \mathrm{ng} / \mathrm{ml}$ recombinant human granulocyte macrophage colony-stimulating factor and $10 \mathrm{ng} / \mathrm{mI}$ recombinant human interleukin-3). The plated cells were cultured in a fully humidified incubator with $5 \% \mathrm{CO}_{2}$ at $3{ }^{\circ} \mathrm{C}$. Colonies were scored after 14 days using an inverted microscope.

Drug sensitivity assay. Chemotherapeutic drug sensitivity was determined using the 3-(4, 5-dimethylthiazol-2-yl)-2, 5-diphenyltetrazolium bromide (MTT) assay kit (Roche, Mannheim, Germany). Briefly, sorted SP and MP cells $\left(3 \times 10^{4}\right.$ per well in 100 $\mu \mathrm{l}$ medium) were seeded in 96-well plates in duplicates. The cells were exposed to chemotherapeutic drugs, namely, $1 \mu \mathrm{M}$ doxorubicin (Sigma Aldrich), $10 \mathrm{nM}$ bortezomib (Millennium Phamaceuticals, Cambridge, MA, USA), $10 \mu \mathrm{M}$ dexamethasone (Sigma Aldrich), and $30 \mu \mathrm{M}$ melphalan (Sigma Aldrich) for $24 \mathrm{~h}$. At the end of the incubation, $10 \mu \mathrm{I}$ of the yellow MTT labeling reagent was added to the culture media. The cells were incubated further for 4 hours at $37^{\circ} \mathrm{C}$, followed by addition of $100 \mu \mathrm{l}$ solubilization solution to dissolve the purple formazan crystals. After standing overnight, absorbance was measured at $562 \mathrm{~nm}$ using a microplate reader (Molecular Devices, San Jose, CA, USA). Drug resistance is presented as percentage viability calculated according to the formula: (absorbance of treated cells)/(absorbance of untreated cells) $\times 100$. 


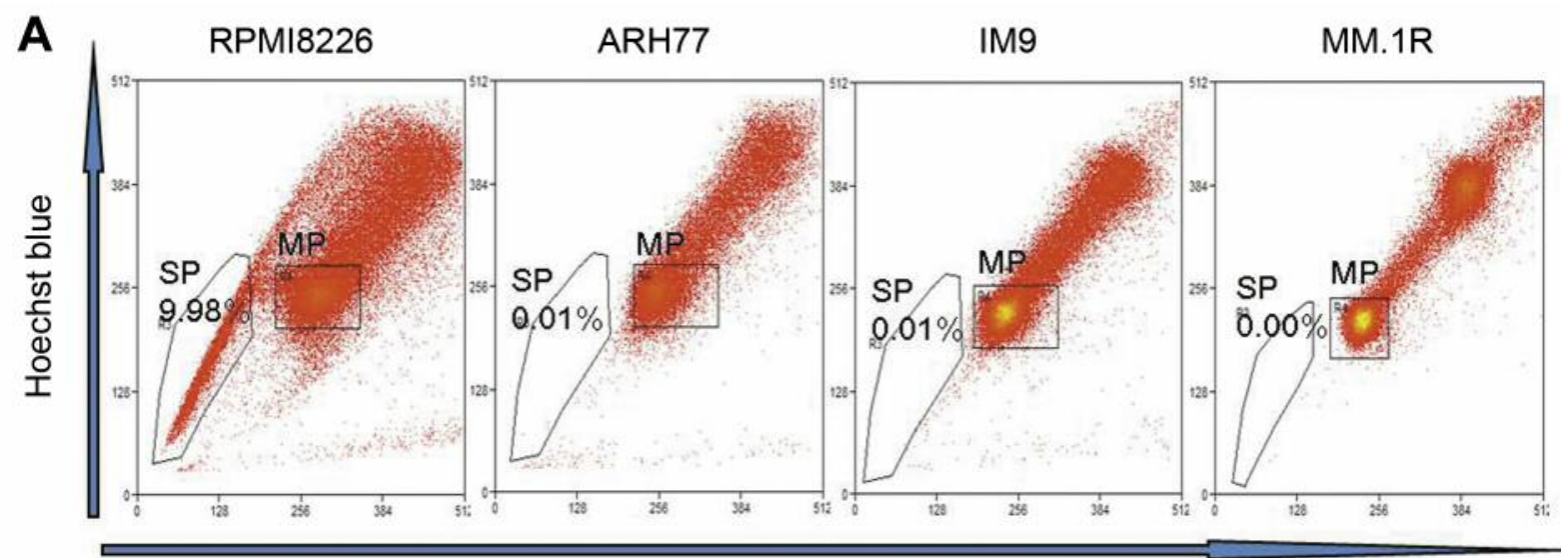

Hoechst red

B
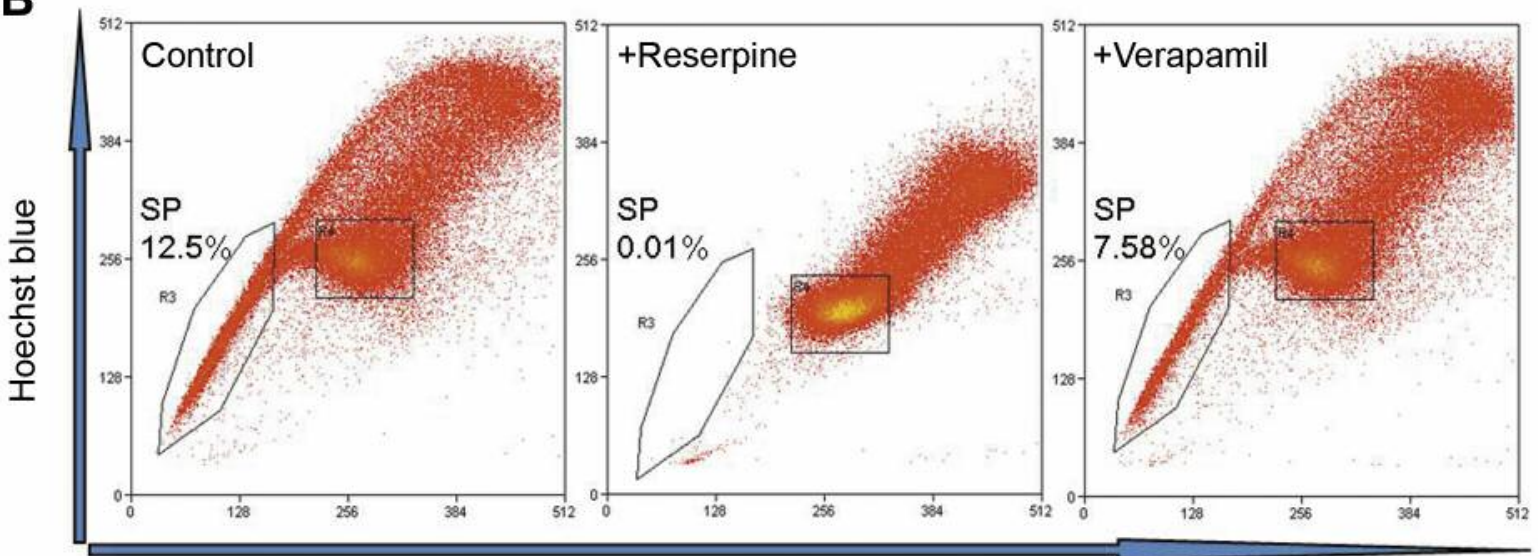

Hoechst red

Figure 1. Characterization of side population (SP) cells in the multiple myeloma (MM) cell lines. A: Representative flow cytometric dot plots of SP analysis in MM cell lines: RPMI8226, ARH77, IM9 and MM.1R. Flow cytometry identified low-fluorescence SP cells in the tail region of the dot plot relative to Hoechst bright-fluorescence cells (MP). B: Dot plots show RPMI8226 cells incubated in Hoechst 33342 alone (left), Hoechst 33342 accumulation in presence of $50 \mu \mathrm{M}$ reserpine (middle), and Hoechst 33342 accumulation in presence of $100 \mu \mathrm{M}$ verapamil (right). Low intracellular accumulation of Hoechst disappeared when cells were treated with ATP-binding cassette transporter inhibitors such as reserpine or verapamil. Similar results were obtained by three independent experiments.

Statistical analysis. Statistical comparisons were performed using the Student's $t$-test. $p$-Values of less than 0.05 were considered to be statistically significant.

\section{Results}

Identification of SP cells based on Hoechst 33342 dye exclusion. The SP cells were detected based on their ability to exclude Hoechst 33342 dye. Flow cytometry identified low-fluorescent (SP) cells in the bottom-left corner of the cytogram relative to the Hoechst-positive bright-fluorescent cells (MP). SP analysis was performed of four human MM cell lines (RPMI8226, ARH77, IM9, and MM.1R). ARH77,
IM9, and MM.1R contained a negligible SP fraction $(<0.1 \%)$, which was not enough for further studies. However, the RPMI8226 cell line had a higher SP fraction (10-18\%). Based on these results, we selected the RPMI8226 cell line for use in further experiments (Figure 1A). The SP phenotype is mediated by $\mathrm{ABC}$ transporter proteins. This was confirmed as the SP population decreased significantly when the cells were pretreated with ABC transporter inhibitor verapamil $(100 \mu \mathrm{mol} / \mathrm{l})$ or reserpine $(50 \mu \mathrm{mol} / \mathrm{l})$. The RPMI8226 cell line contained $12.5 \%$ SP cells. After the cells were pretreated with reserpine and verapamil, this percentage decreased to $0.01 \%$ and $7.58 \%$, respectively (Figure 1B). 


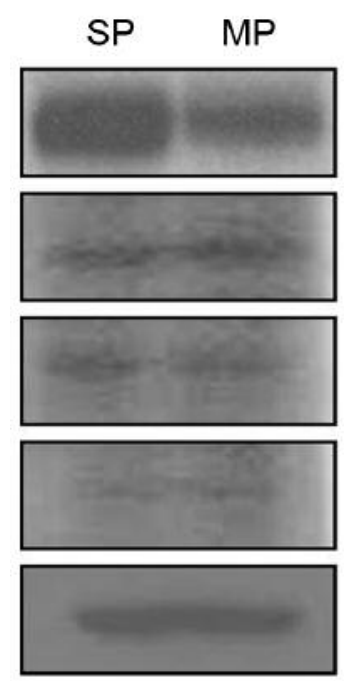

ABCG2

OCT4

Sox2

NANOG

$\beta$-Actin

Figure 2. Expression of cancer stem cell markers in sorted side population (SP) and main population (MP) cells from RPMI8226. Thirty micrograms of whole-cell lysates were resolved by sodium dodecyl sulfate-polyacrylamide gel electrophoresis and subjected to western blotting with antibodies against ATP-binding cassette subfamily $G$ member 2 (ABCG2), octamer-binding transcription factor 4 (OCT4), sex determining region Y-box 2 (SOX2), and NANOG. Equal loading was determined by $\beta$-actin. SP cells expressed more ABCG2 than MP cells. Similar results were obtained in three independent experiments.

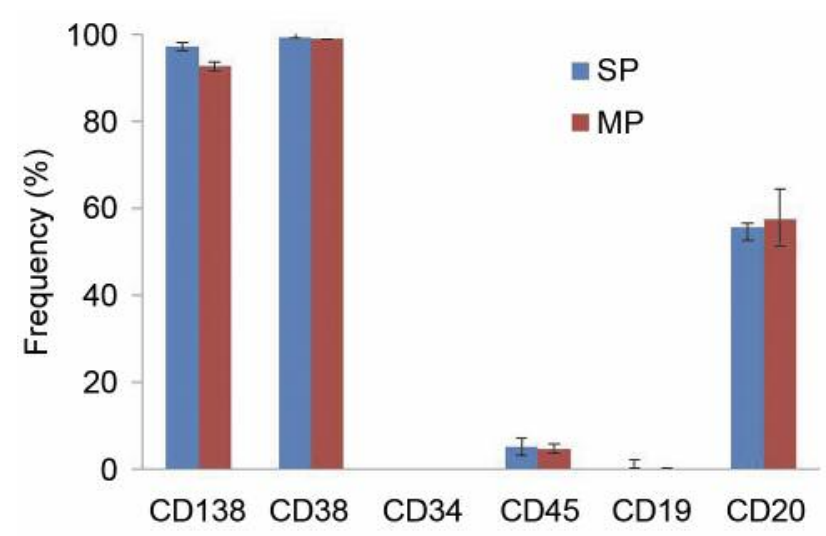

Figure 3. Characterization of the phenotype of sorted side population (SP) and main population (MP) cells from RPMI8226 cells. The expression of multiply myeloma-related cell surface antigens [cluster of differentiation (CD)138, CD38, CD34, CD45, CD19, and CD20] was analyzed by flow cytometry and did not differ between SP and MP cells. Similar results were obtained by three independent experiments.

Expression of stem cell markers in SP cells. The SP phenotype in hematopoietic stem cells is mediated primarily by the cell surface-associated ABCG2 transporter. ABCG2 contributes to resistance to various chemotherapeutic agents
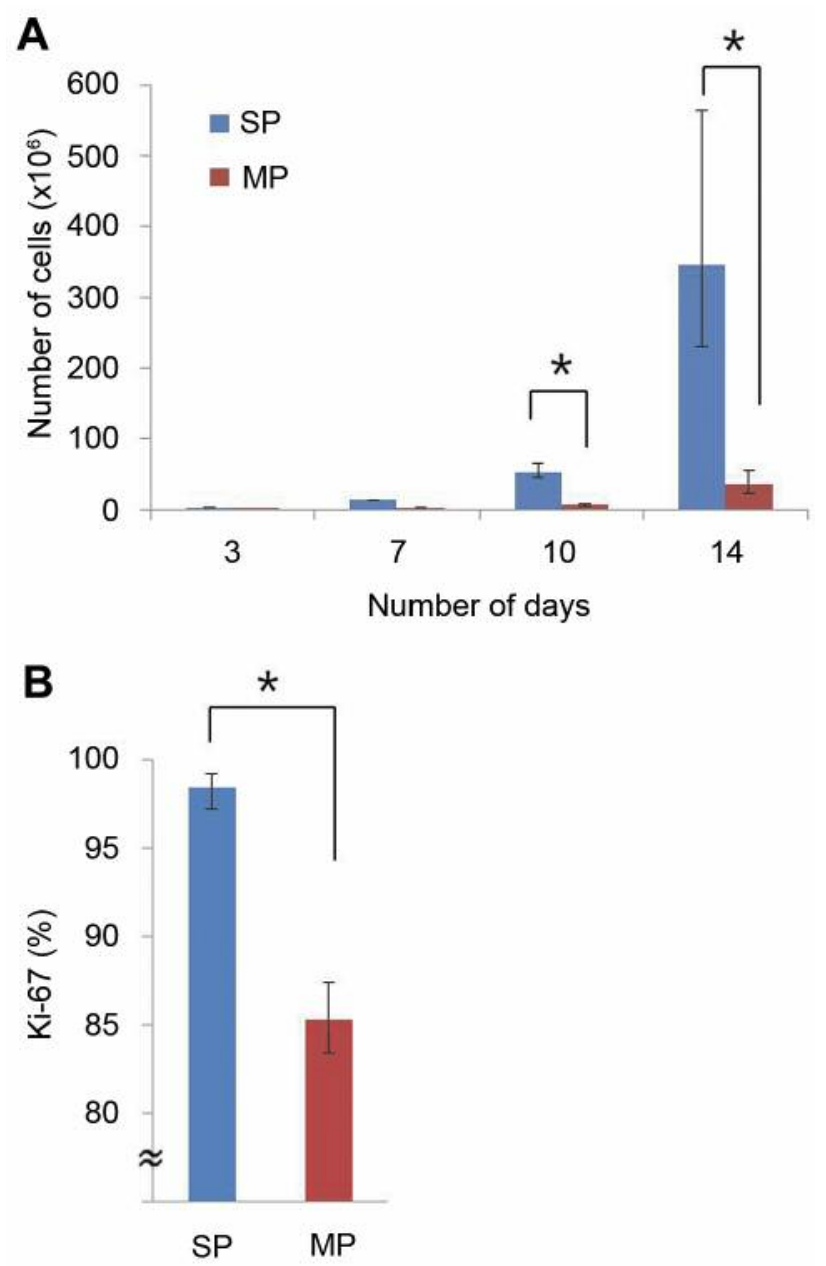

Figure 4. Comparison of cell proliferative activity for sorted side population (SP) and main population (MP) cells. A: Comparison of cell growth was observed using the trypan blue exclusion assay. B: Expression of Ki-67 was assessed by flow cytometry. SP cells expressed higher levels of the proliferative marker Ki-67 than MP cells. Columns represent the average for three independent experiments with similar results. *Significantly different at $p<0.05$.

in the stem cell population. Therefore, we analyzed the ABCG2 levels in the sorted SP and MP cells from the RPMI8226 cell line. In addition, we determined the levels of OCT4, SOX2, and NANOG, known cancer stem cell markers. The sorted SP cells expressed more ABCG2 than did the MP cells (Figure 2). However, the levels of OCT4, SOX2, and NANOG did not appear to differ between SP and MP cells (Figure 2).

Characterization of MM-related cell surface antigens in SP cells. MM cell lines and clinical specimens are characterized by the presence of malignant plasma cells expressing CD138, a surface antigen. During normal B-cell 


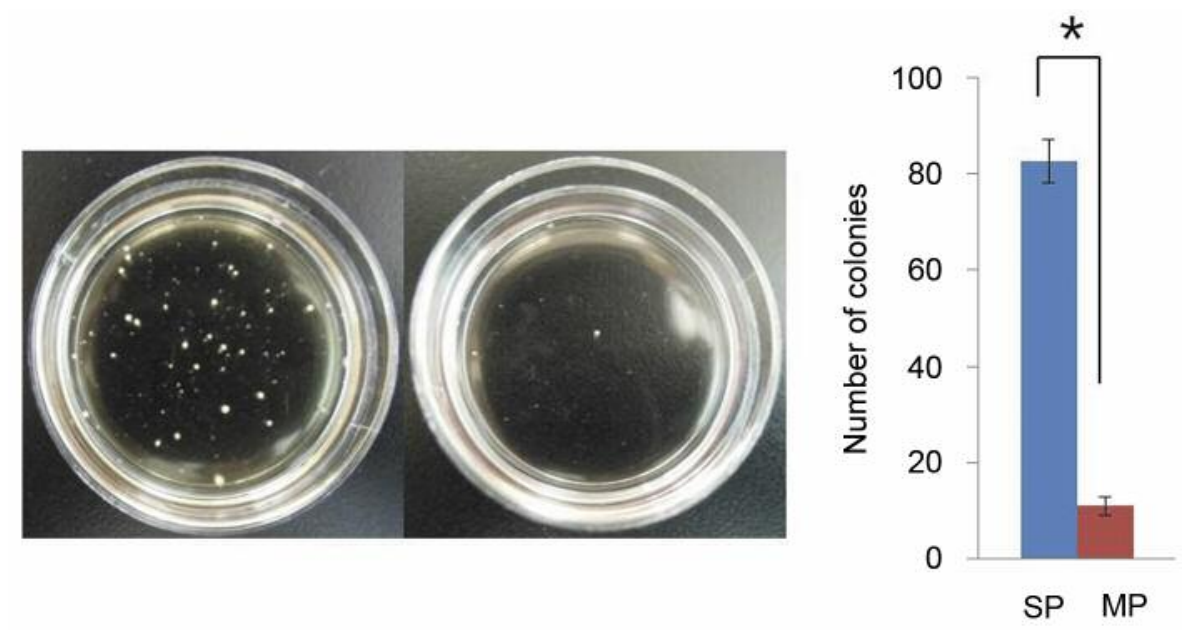

Figure 5. Clonogenicity of sorted side population (SP) and main population (MP) cells from RPMI8226. The number of colonies was assessed at 14 days. Columns represent the average for three independent experiments with similar results. *Significantly different at $p<0.05$.

development, CD138 is specifically expressed on the terminally differentiated normal plasma cells and it is absent from highly proliferative normal plasma blasts and all earlier B-cell stages. Assuming that clonogenic B-cells should lack CD138 expression if they represent putative MM stem cells, we examined the expression of CD138 and other surface antigens (CD38, CD34, CD45, CD19, and CD20) in the SP and MP cells obtained from the RPMI8226 cell line. The expression of the MM-related cell surface antigens, including CD138, did not significantly differ between SP and MP cells (Figure 3).

Cell growth and colony formation of SP cells. Stem-like cells have been reported to possess high proliferative potential. To determine whether the sorted SP cells from RPMI8226 have stem-like cell properties, we first cultured the sorted SP and MP cells separately under the same conditions. We observed cell growth of the sorted SP and MP cells after 3, 7, 10, and 14 days of culture using trypan blue exclusion assay. The sorted SP cells had significantly higher proliferative potential compared to the MP cells. The average number of sorted SP and sorted MP cells after 14 days of culture significantly increased (Figure 4A, $p<0.05$ ). The SP cells expressed higher levels of $\mathrm{Ki}-67$, which indicated that the SP cells proliferated more than the MP cells (Figure $4 \mathrm{~B}, p<0.05$ ). To assess the clonogenicity of the SP cells, we performed colony-forming assays with SP and MP cells sorted from RPMI8226. The assay was performed in methylcellulose for 14 days. As shown in Figure 5, the SP cells generated more colonies than did the MP cells, and the average colony count of SP cells was significantly higher than that of MP cells (almost 8-fold, $p<0.05$ ).

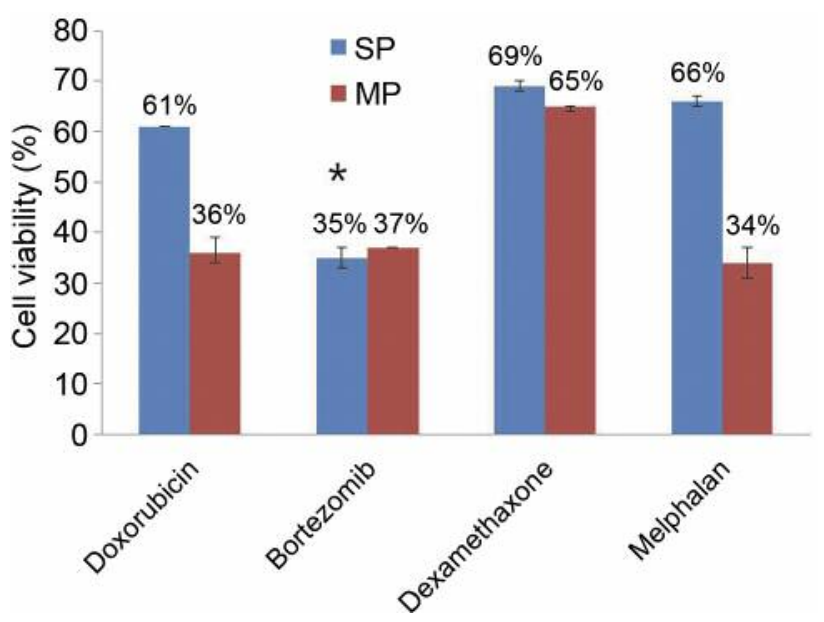

Figure 6. Drug sensitivity of sorted side population (SP) and main population (MP) cells from RPMI8226. The y-axis shows the viability of SP and MP cells following treatment with the indicated drugs for $24 \mathrm{~h}$. Cell viability was assessed by MTT assay. Columns represent the average for three independent experiments with similar results. Only bortezomib treatment significantly reduced cell viability of SP cells compared to doxorubicin, melpalan, and dexamethasone $(* p<0.05)$.

Drug sensitivity of SP cells. To determine whether the SP cells are associated with resistance to chemotherapy, we treated the SP and MP cells sorted from RPMI8226 with four clinically active agents: doxorubicin $(1 \mu \mathrm{M})$, bortezomib (10 $\mathrm{nM})$, dexamethasone $(10 \mu \mathrm{M})$, and melphalan $(30 \mu \mathrm{M})$. After treatment with these drugs for 24 hours, we determined cell viability using the MTT assay. The SP cells were highly resistant to chemotherapeutic drugs when compared to the 
MP cells. When treated with doxorubicin and melphalan, the viability of MP cells was practically half that of SP cells (Figure 6). Dexamethasone treatment elicited negligible difference in cell viability between the SP and MP cells. In contrast, bortezomib treatment significantly reduced cell viability of both SP and MP cells (Figure 6, $p<0.05$ ). Bortezomib was the only agent that significantly affected proliferation of both SP and MP cells.

\section{Discussion}

Human ABCG2 can efflux Hoechst 33342, that can reflect an SP phenotype (16). Previous studies addressing the mechanism of Hoechst exclusion from cells have relied on a correlation between dye exclusion and high transporter expression in cell lines that were selected for high drug resistance (9). The SP cells, which are a small subset of cells found in MM, were isolated from MM cell lines on similar principles. This result is in agreement with the results of studies conducted to confirm the presence of SP cells in other diseases such as lymphoma, lung cancer, and myeloma $(17,18)$. The SP cells isolated from human cancer exhibit stem-like properties, including high tumorigenic activity and chemoresistance.

In this study, the SP cells showed increasing cell growth during culture and greater ability to form colonies than the MP cells. The average colony count of SP cells was significantly higher than that of MP cells. In addition, the SP cells expressed higher levels of Ki-67 than the MP cells. These findings suggest that the SP cells form a highly tumorigenic subpopulation in MM, a quiescent type of cancer $(19,20)$. ABCG2 contributes largely to the properties of SP cells $(21,22)$. We observed that the sorted SP cells expressed more ABCG2 than the MP cells. Therefore, we surmised that SP cells may share many functional properties of myeloma stem cells, and examined the expression of certain known cancer stem cell markers OCT4, SOX2, and NANOG in SP cells. However, OCT4, SOX2, and NANOG expression did not differ between the SP and MP cells. This means the SP cell characteristics are not completely identical to those of myeloma stem cells.

Several reports have revealed features of myeloma stem cells; however, these results are generally inconsistent. These studies evaluated whether specific surface markers are expressed selectively on MM stem cells but not on the bulk tumor cells. Normal plasma cells arise from the B-cell maturation, and the MM plasma cells were identified to be $\mathrm{CD} 34^{-} \mathrm{CD} 19^{-} \mathrm{CD} 138^{+}$by FACS analysis. CD138 is the most specific marker of MM plasma cells. The first hypothesis posits that if clonogenic B-cells might express CD19 or CD20 if they represent the putative MM stem cells (3). The second hypothesis posits that MM stem cells should not express CD138 $(3,23)$. In our study, the expression of cell-surface antigens, including CD19, CD20, CD138, was not different between SP and MP cells.
In 2004, Matsui et al. reported that the human MM cell lines contained a small CD138- subpopulation $(<5 \%)$ with greater clonogenic potential than the $\mathrm{CD} 138^{+}$plasma cells. Furthermore, the CD138- MM cell lines phenotypically resembled post-germinal center B-cells, and their clonogenic growth was inhibited by the anti-CD20 monoclonal antibody rituximab. These data suggested that the $\mathrm{CD} 138^{-} \mathrm{B}$-cells were MM stem cells (3). They later reported that the SP cells from MM cell lines were almost exclusively CD138(>97\%) compared to the bulk population that was $\mathrm{CD} 138^{+}$ (24). In contrast, others reported a lack of correlation between SP cells and the CD138 $8^{-/+}$subpopulation in MM cell lines (25). Chiron et al. reported that clonogenic myeloma cells were $\mathrm{CD} 138^{+} \mathrm{CD} 20^{-}$cells (25). However, these findings do not agree with the results of Matusi et al. Thus, the origin and identity of clonogenic myeloma cells (B-cells or non-B-cells) (i.e. whether the clonogenic myeloma cells are identical to SP cells) is still a matter of debate. In addition, we did not establish the existence of any phenotypic differences between SP cells and MP cells.

The SP cells might contribute to the aggressive behavior of certain tumor types and the drug-resistant phenotype of other tumors because of its tumorigenic potential and the presence of the ABCG2 cell-surface drug transporter (21, 22). To determine whether the SP cells are associated with resistance to chemotherapy, we treated sorted SP and MP cells with four clinically active agents, namely, doxorubicin, bortezomib, dexamethasone, and melphalan. The viability of the SP cells was higher than that of the MP cells except in the presence of bortezomib. Importantly, bortezomib treatment significantly reduced the viability of both SP and MP cells (Figure 6). Jakubikova et al. reported that lenalidomide, but not thalidomide treatment, inhibited the proliferation of the SP cells of MM cell lines (13). They suggested that lenalidomide might target SP cells with clonogeneic potential, providing the framework for development of new treatment strategies targeting putative MM stem/tumor-initiating cells. However, they did not evaluate the sensitivity of SP cells to proteasome inhibitors. Finally, we showed that the proliferation of SP cells was inhibited by bortezomib treatment in MM cell lines, but not by cytotoxic agents and dexamethasone. We expect that bortezomib will be effective against SP cells of MM.

\section{Conclusion}

We demonstrated the presence of SP cells in human MM cell lines. Although it is difficult to conclusively ascertain whether SP cells are similar/identical to myeloma stem cells, the SP cells showed superior colony-forming ability and higher resistance to chemotherapeutic agents compared to the MP cells. Only bortezomib significantly inhibited proliferation of both SP and MP cells. Thus, bortezomib might target SP cells. 


\section{Conflicts of Interest}

All the Authors declare no potential conflicts of interest in regard to this study.

\section{Acknowledgements}

This work was supported, in part, by the Soonchunhyang University Research Fund.

\section{References}

1 Kyle RA and Rajkumar SV: Multiple myeloma. N Engl J Med 351(18): 1860-1873, 2004.

2 Huff CA and Matsui W: Multiple myeloma cancer stem cells. J Clin Oncol 26(17): 2895-2900, 2008.

3 Matsui W, Huff CA, Wang Q, Malehorn MT, Barber J, Tanhehco Y, Smith BD, Civin CI and Jones RJ: Characterization of clonogenic multiple myeloma cells. Blood 103(6): 2332-2336, 2004.

4 Epstein J: Myeloma stem cell phenotype. Implications for treatment. Hematol Oncol Clin North Am 11(1): 43-49, 1997.

5 Berenson JR, Vescio RA and Said J: Multiple myeloma: The cells of origin-a two-way street. Leukemia 12(2): 121-127, 1998.

6 Mitterer M, Oduncu F, Lanthaler AJ, Drexler E, Amaddii G, Fabris P, Emmerich B, Coser P and Straka C: The relationship between monoclonal myeloma precursor B-cells in the peripheral blood stem cell harvests and the clinical response of multiple myeloma patients. Br J Haematol 106(3): 737-743, 1999.

7 Davies FE, Rawstron AC, Owen RG and Morgan GJ: Controversies surrounding the clonogenic origin of multiple myeloma. Br J Haematol 110(1): 240-241, 2000.

8 Hosen N, Matsuoka Y, Kishida S, Nakata J, Mizutani Y, Hasegawa K, Mugitani A, Ichihara H, Aoyama Y, Nishida S, Tsuboi A, Fujiki F, Tatsumi N, Nakajima H, Hino M, Kimura T, Yata K, Abe M, Oka Y, Oji Y, Kumanogoh A and Sugiyama H: CD138-negative clonogenic cells are plasma cells but not B-cells in some multiple myeloma patients. Leukemia 26(9): 2135-2141, 2012.

9 Ho MM, Ng AV, Lam S and Hung JY: Side population in human lung cancer cell lines and tumors is enriched with stem-like cancer cells. Cancer Res 67(10): 4827-4833, 2007.

10 Li C, Heidt DG, Dalerba P, Burant CF, Zhang L, Adsay V, Wicha M, Clarke MF and Simeone DM: Identification of pancreatic cancer stem cells. Cancer Res 67(3): 1030-1037, 2007.

11 Ricci-Vitiani L, Lombardi DG, Pilozzi E, Biffoni M, Todaro M, Peschle $\mathrm{C}$ and De Maria R: Identification and expansion of human colon-cancer-initiating cells. Nature 445(7123): 111-115, 2007.

12 Challen GA and Little MH: A side order of stem cells: The SP phenotype. Stem Cells 24(1): 3-12, 2006.

13 Jakubikova J, Adamia S, Kost-Alimova M, Klippel S, Cervi D, Daley JF, Cholujova D, Kong SY, Leiba M, Blotta S, Ooi M, Delmore J, Laubach J, Richardson PG, Sedlak J, Anderson KC and Mitsiades CS: Lenalidomide targets clonogenic side population in multiple myeloma: Pathophysiologic and clinical implications. Blood 117(17): 4409-4419, 2011.
14 Nara M, Teshima K, Watanabe A, Ito M, Iwamoto K, Kitabayashi A, Kume M, Hatano Y, Takahashi N, Iida S, Sawada $\mathrm{K}$ and Tagawa $\mathrm{H}$ : Bortezomib reduces the tumorigenicity of multiple myeloma via down-regulation of upregulated targets in clonogenic side population cells. PLoS One 8(3): e56954, 2013.

15 Du J, Liu S, He J, Liu X, Qu Y, Yan W, Fan J, Li R, Xi H, Fu W, Zhang C, Yang J and Hou J: MicroRNA-451 regulates stemness of side population cells via $\mathrm{PI} 3 \mathrm{~K} / \mathrm{AKT} / \mathrm{mTOR}$ signaling pathway in multiple myeloma. Oncotarget 6(17): 14993-15007, 2015.

16 Goodell MA, Brose K, Paradis G, Conner AS and Mulligan RC: Isolation and functional properties of murine hematopoietic stem cells that are replicating in vivo. J Exp Med 183(4): 1797-1806, 1996.

17 Lee MR, Ju HJ, Kim BS, Ko YH, Kim WS and Kim SJ: Isolation of side population cells in B-cell non-Hodgkin's lymphomas. Acta Haematol 129(1): 10-17, 2013.

18 Dai Y, Liu S, Zhang WQ, Yang YL, Hang P, Wang H, Cheng L, Hsu PC, Wang YC, Xu Z, Jablons DM and You L: YAP1 regulates $\mathrm{ABCG} 2$ and cancer cell side population in human lung cancer cells. Oncotarget 8(3): 4096-4109, 2017.

19 Drewinko B, Alexanian R, Boyer H, Barlogie B and Rubinow SI: The growth fraction of human myeloma cells. Blood 57(2): 333-338, 1981.

20 Hamburger A and Salmon SE: Primary bioassay of human myeloma stem cells. J Clin Invest 60(4): 846-854, 1977.

21 Salcido CD, Larochelle A, Taylor BJ, Dunbar CE and Varticovski L: Molecular characterisation of side population cells with cancer stem cell-like characteristics in small-cell lung cancer. Br J Cancer 102(11): 1636-1644, 2010.

22 Summer R, Kotton DN, Sun X, Ma B, Fitzsimmons K and Fine A: Side population cells and BCRP1 expression in lung. Am J Physiol Lung Cell Mol Physiol 285(1): L97-104, 2003.

23 Asosingh K, De Raeve H, Van Riet I, Van Camp B and Vanderkerken K: Multiple myeloma tumor progression in the 5T2MM murine model is a multistage and dynamic process of differentiation, proliferation, invasion, and apoptosis. Blood 101(8): 3136-3141, 2003.

24 Matsui W, Wang Q, Barber JP, Brennan S, Smith BD, Borrello I, McNiece I, Lin L, Ambinder RF, Peacock C, Watkins DN, Huff CA and Jones RJ: Clonogenic multiple myeloma progenitors, stem cell properties, and drug resistance. Cancer Res 68(1): 190-197, 2008.

25 Chiron D, Surget S, Maiga S, Bataille R, Moreau P, Le Gouill $\mathrm{S}$, Amiot M and Pellat-Deceunynck C: The peripheral CD138+ population but not the CD138- population contains myeloma clonogenic cells in plasma cell leukaemia patients. $\mathrm{Br} \mathrm{J}$ Haematol 156(5): 679-683, 2012. 